\title{
A novel mechanism whereby BRCA1/1a/1b fine tunes the dynamic complex interplay between SUMO-dependent/independent activities of Ubc9 on E2-induced ER $\alpha$ activation/repression and degradation in breast cancer cells
}

\author{
J. XU*, T. WATKINS*, A. REDDY, E.S.P.REDDY and V.N. RAO \\ Cancer Biology Program, Department of OB/GYN, Morehouse School of Medicine, \\ Georgia Cancer Center for Excellence, Grady Health System, Atlanta, GA 30303, USA
}

Received November 5, 2008; Accepted December 19, 2008

DOI: 10.3892/ijo_00000220

\begin{abstract}
BRCA1 dysfunction is associated with hormoneresponsive cancers. We have identified a consensus SUMO modification site in the amino-terminal region of BRCA1/1a/1b proteins and the mutation in this potential SUMO acceptor site (K 109 to $\mathrm{R}$ ) impaired their ability to bind and repress ligand-dependent $\mathrm{ER} \alpha$ transcriptional activity in breast cancer cells. Furthermore, we have found SUMO E2-conjugating enzyme Ubc9 to bind BRCA1 proteins. We have mapped BRCA1 [within amino acids (aa) 1-182] as the minimum domain that is sufficient for in vitro binding to Ubc9 as well as for regulating ER $\alpha$ activity. BRCA1 Mutant \#1 (K109 to R) was impaired in its ability to both bind, as well as modulate Ubc9 mediated SUMO-dependent/independent E2-induced $\mathrm{ER} \alpha$ transcriptional activity in breast cancer cells. Similarly, BRCA1 cancer-predisposing mutation (61Cys-Gly) abrogated the ability to both bind Ubc9 as well as inhibit ER $\alpha$ activity suggesting physiological significance. Addition of BRCA1 but not Mutant \#1 to E2-induced ER $\alpha$ in the presence of SUMO-1 and Ubc9 resulted in the degradation of ER $\alpha$ suggesting BRCA1 to be a putative SUMO-1 and Ubc9dependent E3 ubiquitin ligase for ER $\alpha$. This is the first report demonstrating the participation of Ubc9 in BRCA1 E3 ubiquitin ligase mediated degradation of ER $\alpha$. These results suggest a novel function for BRCA1 in regulating the dynamic cycles of SUMO and ubiquitin modifications required for ER $\alpha$ turn over and deregulation of this molecular switch due to lack
\end{abstract}

Correspondence to: Dr Veena N. Rao, Cancer Biology Program, Department of OB/GYN, Morehouse School of Medicine, Georgia Cancer Center for Excellence, RM 10C011, Grady Health System, 80 Jesse Hill Jr. Drive, Atlanta, GA 30303-3031, USA

E-mail:vrao@msm.edu

${ }^{*}$ Contributed equally

Key words: BRCA1/1a/1b, SUMO-1, Ubc9, E3 ubiquitin ligase, estrogen receptor $\alpha$, breast cancers of BRCA1 results in ER $\alpha$-negative/positive breast cancers. This study will help in designing novel BRCA1 function-based targeted treatment for breast cancers.

\section{Introduction}

Inherited mutations of the breast cancer susceptibility gene 1 (BRCA1) confer a high risk for the development of estrogen (E2) dependent breast and ovarian cancers (1-3). The underlying basis for its tissue specific tumor suppressor function remains poorly understood. Multiple naturally occurring isoforms of BRCA1 are present in different tissues with varying expression levels that encode proteins missing all or part of exon 11 (4-7). BRCA1a/p110 and BRCA1b/p100 (8) form two of the four major splice variants present in normal, breast and ovarian cancer cells $(6,9,10)$. Both BRCA1a and BRCA1b differ from BRCA1 in having a deletion of the majority of exon 11 sequences [within amino acids (aa) 263-1365]. BRCA1b has an additional deletion of exons 9 and 10 sequences and they code for $110 / 100 \mathrm{kDa}$ proteins (Fig. 1). BRCA1 and its splice variants are multifunctional proteins that interact with several proteins which regulate a number of biological activities like transcriptional activation/ repression, cell-cycle regulation, growth/tumor suppression, apoptosis, DNA repair, genomic stability, steroid hormone receptor signaling, ubiquitination and sumoylation $(11,12)$ but many of these functions are not tissue specific. Women carrying BRCA1 mutations develop breast tumors that are triple negative (TN) suggesting that hormonal factors play a critical role in the development of these cancers (13). Even though TN breast cancers account for only about $15 \%$ of all breast cancers, they represent a higher percentage of breast cancers arising in African American and younger premenopausal women and currently there are no targeted therapies available for these cancers (14). There is significant overlap between TN breast cancers and BRCA1 associated breast cancers both histologically as well as transcriptionally, which suggests that dysfunction in the BRCA1 pathway may be responsible for the development of these cancers $(15,16)$. Estrogen receptor $(\mathrm{ER} \alpha)$ signaling has been implicated in the development of BRCA1 associated tissue specific tumorigenesis. Recently we showed BRCA1a to function as a tumor 
suppressor of ER $\alpha$-positive, TN human breast cancers, hormone independent ovarian and prostate cancers both in vitro and in vivo using mouse models (17), but its role in the regulation of ER $\alpha$ activity is not known. Rebbeck et al showed that oophorectomy reduces the risk of breast cancer in BRCA1/BRCA2 mutation carriers (18). BRCA1 inhibits E2 stimulated $\mathrm{ER} \alpha$ activity by direct binding to the AF-2 domain of $\mathrm{ER} \alpha$, by down-regulation of expression of p300 and by interacting with cyclin D1 that inactivates $\mathrm{pRb}$ tumor suppressor gene (19) but none of these mechanisms can explain the similarities between BRCA1 mutation carriers and ER $\alpha$ negative breast cancers.

Post-translational modification of transcriptional factors is important for regulated gene expression. SUMO-1, a 98-amino acid polypeptide, is covalently attached to lysine residues in proteins. Post-translational modification by SUMO has effects on the stability, localization, protein-protein inter-actions and transcriptional regulation (activation or repression). In most cases sumoylation of transcription factors (e.g., HDAC1, p300/CBP, CtBP, STAT1, etc.) and Histone-4 inhibits transcription by promoting recruitment of co-repressors like HDAC complex (12). SUMO-1 also binds to the SUMO binding motifs (20) in BRCA1 and represses BRCA1-mediated transcription in a sumo-independent manner by recruiting HDAC (12). The SBM is different from the SUMO-1 modification consensus sequence $(\Psi-\mathrm{K}-\mathrm{X}-\mathrm{E})$ which is found in SUMO-1 substrate proteins (Table I and Fig. 1). SBM binds SUMO non-covalently but $\Psi-\mathrm{K}-\mathrm{X}-\mathrm{E}$ does not bind to SUMO-1 non-covalently. It appears to bind Ubc9 non-covalently for covalent SUMO attachment. Most SUMO-modified proteins contain the tetra peptide motif $\Psi-\mathrm{K}-\mathrm{X}-\mathrm{D} / \mathrm{E}$ where $\Psi$ is a hydrophobic residue, $\mathrm{K}$ is the lysine conjugated to SUMO, $\mathrm{X}$ is any amino acid, and $\mathrm{D}$ or $\mathrm{E}$ is an acidic residue (21). A wide variety of proteins are sumoylated (Table I). ER $\alpha$ is a nuclear transcription factor that undergoes various types of post-translational modifications like phosphorylation, acetylation, ubiquitination, methylation, and sumoylation (22). $\mathrm{ER} \alpha$ is sumoylated at conserved lysine residues within the hinge region only in the presence of E2 (22). PIASI, PIAS3 act as E3 ligases and Ubc9 as E2 SUMO-conjugating enzyme for $\mathrm{ER} \alpha$ sumoylation and also modulate $\mathrm{ER} \alpha$ transcription independent of SUMO-1 conjugation activity. Mutations that abrogate sumoylation impair ER $\alpha$ dependent transcriptional activation but not its subcellular localization. Ubc9 is the only mammalian E2 conjugating enzyme that is essential for sumoylation. The mutant C93S Ubc9, which prevents SUMO-1 conjugation by preventing the formation of thiolester bond between SUMO-1 and Ubc9, still functions as a coactivator for nuclear receptors (23). Thus there can be sumodependent transcriptional activation or repression and sumoindependent transcriptional activation or repression of promoters. A role for post-translational modifications in targeting ER $\alpha$ for degradation is poorly understood. A direct correlation has been observed by some between the rate of $\mathrm{ER} \alpha$ degradation and its transcriptional activation and treatment of cells with proteosome inhibitor MG132, impaired ER $\alpha$ transcription (24). Furthermore ER $\alpha$ is ubiquitylated after the first round of transcription which may be needed for subsequent E2-mediated ER $\alpha$ transcription. ER $\alpha$ thus cycles on and off the promoter as long as E2 is present (25-28). SUMO-1 was found to suppress BRCA1 mediated transcription of Gadd45 $\alpha$, $\mathrm{p} 27^{\mathrm{KIP} 1}$ and $\mathrm{p} 21^{\mathrm{WAF} / \mathrm{CIP} 1}$ via modulation of promoter occupancy (12). The only known enzymatic activity that is associated with BRCA1 is its E3 ubiquitin ligase activity, and recently $\mathrm{ER} \alpha$ was shown to be a putative substrate for this BRCA1/ BARD1 ubiquitin ligase activity (29). Deleterious BRCA1 RING-finger domain mutations eliminated the ubiquitin ligase activity, but did not eliminate its auto ubiquitination activity suggesting a possible link between tumor suppressor function of BRCA1 and its E3 ligase activity (30). However all these studies do not uncover the paradox as to why impairment of this E3-ligase activity contributes to ERnegative breast cancers.

In this report we have identified an SCS (SUMO-1 modification consensus sequence) in the conserved amino terminal RING finger domain of BRCA1/1a/1b proteins and mutation in this SUMO acceptor site and cancer-predisposing mutation C61G impaired their ability to both interact with ER $\alpha$ and SUMO-E2-conjugating enzyme Ubc9 as well as modulate Ubc9 mediated SUMO-dependent/independent E2-induced $\mathrm{ER} \alpha$ transcriptional activation in breast cancer cells. Ubc9 is a new player in the E2-induced ER $\alpha$ regulation by BRCA1 proteins. Our results suggest BRCA1/1a/1b proteins but not K109 to R mutant to be putative SUMO-1 and Ubc9-dependent E3 ubiquitin ligases that bind and recruit the E2 ubiquitin conjugating enzyme Ubc9 to ER $\alpha$ and facilitate the ubiquitination and proteosome dependent degradation of ER $\alpha$ in an estrogen-dependent manner. These findings suggest a novel mechanism whereby BRCA1 fine tunes the dynamic interplay between SUMO dependent/independent activities of Ubc9 on E2-induced ER $\alpha$ regulation and dysfunction in this activation-repression switch due to lack of BRCA1 results in ER-negative and ER-positive breast cancers. The results from these studies will shed some light on the enigma as to why BRCA1 dysfunction leads to ER-negative breast cancers.

\section{Materials and methods}

Cell culture. MCF-7, T47 D and COS-1 cells were obtained from American Type Culture Collection (Rockville, MD, USA). MCF-7 cells were cultivated in MEM with $10 \%$ fetal bovine serum, $1 \%$ penicillin and streptomycin, $10 \mathrm{mg} / \mathrm{ml}$ insulin. T47D were grown in DMEM with $10 \%$ fetal bovine serum, $0.2 \mathrm{U} / \mathrm{ml}$ insulin, $1 \%$ penicillin streptomycin and $0.15 \%$ sodium bicarbonate.

Expression constructs. Expression plasmid encoding full-length BRCA1 in pcDNA3, pSG5-ER $\alpha$ and ERE-TK-luciferase reporter, pcDNA3 Ubc9, pcDNA3 Ubc9 C93S both V5tagged and pcDNA3 6x His/SUMO-1, and pGEX-Ubc9 were generously provided by Dr Michael Erdos (National Human Genome Research Institute, Bethesda, MD, USA), Professor M.G. Parker (London, UK), Professor Ron Hay and Dr Ellis Jaffrey (University of Dundee, UK), and Dr Michael J. Mutanis (Johns Hopkins University), respectively. The pCDNA3 BRCA1a and pCDNA3 BRCA1b plasmids have been described previously (8). cDNAs for BRCA1 1-182 was constructed by PCR cloning and sub-cloning into the pCMV-Tag 2 vector (Stratagene, La Jolla, CA, USA). This 
a

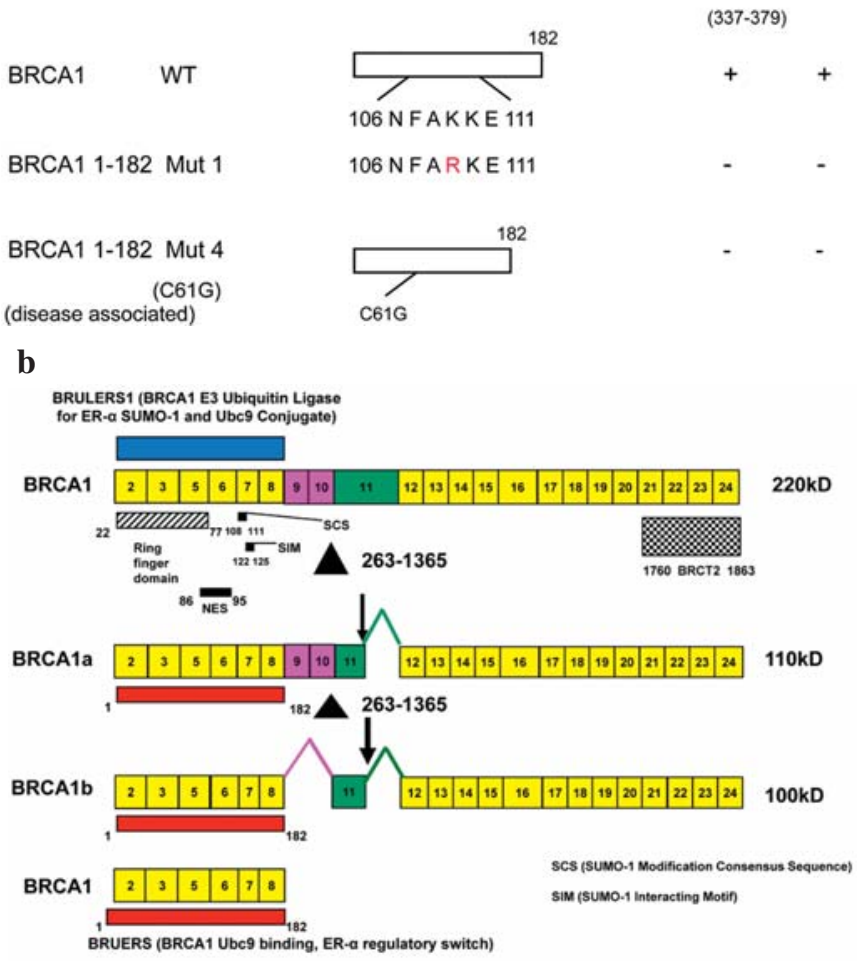

Figure 1. (a) A 182-amino acid domain near the amino-terminus of BRCA1/1a/1b but not Mut\#1 and disease associated C61G Mut\#4 mediates $\mathrm{ER} \alpha$ and Ubc 9 binding. (b) Structure of BRCA1/1a/1b showing the putative domain for BRCA1 E3 ubiquitin ligase for ER $\alpha$ SUMO-1 and Ubc9 conjugate (BRULERS1), sumo-modification consensus sequence (SCS), nuclear export sequence (NES), sumo-1 interacting motif (SIM) and BRCA1 Ubc9 binding $\mathrm{ER} \alpha$ regulatory switch (BRUERS).

vector allows expression of BRCA1 protein with an $\mathrm{N}$ terminal FLAG sequence. BRCA1, 1a, 1b, BRCA1 1-182 point mutants 1 , and 4 (see Fig. $1 \mathrm{~b}$ and text) were constructed using Quick change site directed Mutagenesis kit (Stratagene).
GST-pull down assays. GST-Ubc9 and GST-ER $\alpha$ (337-379) were expressed and purified from $E$. coli as described previously (8). For in vitro protein binding assay $35 \mathrm{~S}$ methionine-labeled in vitro translated full length BRCA1, BRCA1a (31), BRCA1b (31), BRCA1 Mut\#1, BRCA1 Mut\#4, BRCA1 1-182, BRCA1 1-182 Mut\#1 and BRCA1 1-182 Mut 4 proteins were diluted in binding buffer [20 mM Tris- HCI ( $\mathrm{pH} 7.5$ ), $150 \mathrm{mM} \mathrm{NaCl}, 0.1 \%$ Tween-20]. The proteins were precleared with GSH beads for $30 \mathrm{~min}$ and then incubated with GST or various GST-ER (337-379) proteins beads for $2 \mathrm{~h}$ at $4^{\circ} \mathrm{C}$. The beads were then washed three times with binding buffer followed by elution of the bound proteins with SDS sample buffer and loaded on a $15 \%$ SDS-PAGE. The gels were fixed, treated with enhance, dried and exposed to X-ray film and scanned using a Fuji Bio-Imaging analyzer. Competition studies using Ubc9 and Ubc9C93S as competitors were performed as described above except that $50 \mu \mathrm{l}$ of BRCA 1-182 translated proteins were pre-incubated with increasing amounts of $(40,60$, and $80 \mu \mathrm{l}$ ) of in vitro translated Ubc9 and Ubc9C93S proteins before the addition of GST-ER (337-379) beads. The gels were fixed, treated with enhance, dried, exposed and scanned using a phospho-Imager.

Immunofluorescence staining. To study the co-localization of endogenous and exogenous BRCA1 and BRCA1a with Ubc9 in vivo, MCF-7 cells were cultured on glass cover slips in 6-well plates overnight. Sub confluent cells were either used as such or transfected overnight with the indicated expression plasmid (1.5 $\mu \mathrm{g}$ BRCA1 or BRCA1a) using Lipofectamine and incubated in fresh medium for $24 \mathrm{~h}$. Cells were then washed with PBS, fixed in ice cold methanol for $5 \mathrm{~min}$, and then washed with PBS. The cells were then blocked with $10 \%$ bovine serum albumin in PBS for $30 \mathrm{~min}$ at room temperature, followed by incubation with Image-iT FX signal enhancer (I36933, Invitrogen, Carlsbad, CA, USA) for $30 \mathrm{~min}$; incubated with either anti-BRCA1(Ab-1) mouse mAb (MS110) (OP92, EMD Chemicals, Gibbstown, NJ,

Table1. A comparison of the SUMO-1 CS of many known SUMO-1 substrates with BRCA1/1a/1b proteins.

\begin{tabular}{|c|c|c|c|c|c|c|c|c|c|c|c|}
\hline \multirow{2}{*}{$\begin{array}{l}\text { Substrate } \\
\text { RanGAPI }\end{array}$} & \multicolumn{11}{|c|}{ Target site } \\
\hline & $\mathrm{H}$ & M & G & $\mathrm{L}$ & $\mathbf{L}$ & $\mathbf{K}$ & $\mathbf{S}$ & $\mathbf{E}$ & $\mathrm{D}$ & $\mathrm{K}$ & $\mathrm{V}$ \\
\hline PML(1) & $\mathrm{H}$ & Q & W & $\mathrm{F}$ & $\mathbf{L}$ & $\mathbf{K}$ & $\mathbf{H}$ & $\mathbf{E}$ & A & $\mathrm{R}$ & $\mathrm{P}$ \\
\hline PML(2) & $\mathrm{P}$ & $\mathrm{R}$ & $\mathrm{K}$ & $\mathrm{V}$ & I & $\mathbf{K}$ & $\mathbf{M}$ & $\mathbf{E}$ & $\mathrm{S}$ & $\mathrm{E}$ & $\mathrm{E}$ \\
\hline p53 & $\mathrm{K}$ & $\mathrm{K}$ & $\mathrm{L}$ & M & $\mathbf{F}$ & $\mathbf{K}$ & $\mathbf{T}$ & $\mathbf{E}$ & $\mathrm{G}$ & $\mathrm{P}$ & $\mathrm{D}$ \\
\hline $\mathrm{I} \alpha \mathrm{B} \alpha$ & $\mathrm{P}$ & $\mathrm{R}$ & $\mathrm{D}$ & $\mathrm{G}$ & $\mathbf{L}$ & $\mathbf{K}$ & $\mathbf{K}$ & $\mathbf{E}$ & $\mathrm{R}$ & $\mathrm{L}$ & $\mathrm{L}$ \\
\hline c-Jun & $\mathrm{R}$ & $\mathrm{L}$ & Q & A & $\mathbf{L}$ & $\mathbf{K}$ & $\mathbf{E}$ & $\mathbf{E}$ & $\mathrm{P}$ & Q & $\mathrm{T}$ \\
\hline IE2(1) & $\mathrm{M}$ & $\mathrm{L}$ & $\mathrm{P}$ & $\mathrm{L}$ & I & $\mathbf{K}$ & $\mathbf{Q}$ & $\mathbf{E}$ & $\mathrm{D}$ & $\mathrm{I}$ & $\mathrm{K}$ \\
\hline IE2(2) & $\mathrm{K}$ & Q & $\mathrm{E}$ & $\mathrm{D}$ & I & $\mathbf{K}$ & $\mathbf{P}$ & $\mathbf{E}$ & $\mathrm{P}$ & $\mathrm{D}$ & $\mathrm{F}$ \\
\hline HSF2 & $\mathrm{D}$ & $\mathrm{S}$ & G & $\mathrm{I}$ & $\mathbf{V}$ & $\mathbf{K}$ & $\mathbf{Q}$ & $\mathbf{E}$ & $\mathrm{R}$ & $\mathrm{D}$ & $\mathrm{G}$ \\
\hline AR & $\mathrm{P}$ & $\mathrm{H}$ & A & $\mathrm{R}$ & I & $\mathbf{K}$ & $\mathbf{L}$ & $\mathbf{E}$ & $\mathrm{N}$ & $\mathrm{P}$ & $\mathrm{L}$ \\
\hline BRCA1/1a 104 & $\mathrm{~S}$ & $\mathrm{Y}$ & $\mathrm{N}$ & $\mathrm{F}$ & $\mathbf{A}$ & $\mathbf{K}$ & $\mathbf{K}$ & $\mathbf{E}$ & $\mathrm{N}$ & $\mathrm{N}$ & S 114 \\
\hline BRCA1b 104 & $\mathrm{~S}$ & $\mathrm{Y}$ & $\mathrm{N}$ & I & $\mathbf{A}$ & $\mathbf{K}$ & $\mathbf{K}$ & $\mathbf{E}$ & $\mathrm{N}$ & $\mathrm{N}$ & S 114 \\
\hline Consensus & & & & & $\Psi$ & $\mathbf{K}$ & $\mathbf{X}$ & $\mathbf{E}$ & & & \\
\hline
\end{tabular}


USA) or primary mouse monoclonal GFP antibody (sc-9996, Santa Cruz, CA, USA, 1:200 dilution in 1.5\% BSA/PBS) and rabbit polyclonal Ubc9 antibody (sc-10759, Santa Cruz, 1:200 dilution in $1.5 \% \mathrm{BSA} / \mathrm{PBS}$ ) at $25^{\circ} \mathrm{C}$ for $1 \mathrm{~h}$. This was followed by incubation with secondary antibody Alexa Fluor $^{\circledR} 488$ goat anti-mouse IgG $(\mathrm{H}+\mathrm{L})$ highly cross-adsorbed (11034, Invitrogen, 1:200 dilution in 1.5\% BSA/PBS) and Alexa Fluor 568 goat anti-rabbit $\operatorname{IgG}(\mathrm{H}+\mathrm{L})$ highly crossadsorbed (11036, Invitrogen, 1:200 dilution in 1.5\% BSA/PBS) for $50 \mathrm{~min}$. The cells were rinsed with PBS and cover slips mounted in Ultra $\mathrm{Cruz}^{\mathrm{TM}}$ mounting medium with 4,6diamidino-2-phenylindole (DAPI) (sc-24941, Santa Cruz). The stained cells were examined using an Olympus 1 X71 microscope, equipped with $100 \mathrm{X} / 1.25$ oil ph immersion objectives. Composite filter cubes were used for the FITC, DAPI and TRITC fluorescence, respectively. The pictures were captured with a Moticam 3000 camera.

Co-immunoprecipitation. To study the association of Ubc9 with BRCA1a protein proliferating COS-1 cells at $\sim 70 \%$ of confluence in 100-mm petridishes were transfected for $48 \mathrm{~h}$ with (18 $\mu \mathrm{g}$ of plasmid DNA/dish), using FuGENE 6. The cells were harvested and whole-cell extracts was prepared using ER $\alpha$ RIPA buffer as described (22). The extracts were incubated overnight at $4^{\circ} \mathrm{C}$ with $10 \mu 1$ of anti-FLAG M2 affinity gel (Sigma Aldrich Inc.). After low speed centrifugation, the agarose beads were washed with PBS and the pellet was suspended in boiling SDS sample buffer and subjected to 4-20\% SDS-PAGE followed by Western blot analysis using anti-Flag/anti-V5 antibodies.

Western blot analysis. For Western blot analysis total protein lysates were electrophoresed on a 4-20\% SDS polyacrylamide gradient gel. The proteins were transferred onto nitrocellulose membranes and probed with anti-Flag (M2 mouse monoclonal (Sigma, 1:500 dilution) or anti-V5 mouse monoclonal (Invitrogen, 1:2000 dilution) antibodies. Antibody binding was detected by using the enhanced chemiluminescence system (ECL), with Page Ruler ${ }^{\mathrm{TM}}$ plus pre-stained protein ladder (Fermentas) as molecular weight standards.

Assay for the effect of E2 on ERa transcriptional activation. T47D cells $\left(5 \times 10^{5}\right)$ were plated in 6 -well plates for $24 \mathrm{~h}$. The cells were washed twice in PBS followed by the addition of phenol-red free DMEM containing 5\% charcoal-stripped FBS for $48 \mathrm{~h}$ before transfection. The cells were transfected with $1 \mu \mathrm{g}$ of each of the indicated plasmids (with a total of $2.5 \mu$ g DNA) using Lipofectamine 2000 (Life Technology, USA). The cells were induced with $1 \mu \mathrm{M}$ of E2 $4 \mathrm{~h}$ after transfections, harvested after an additional $40 \mathrm{~h}$ and assayed for luciferase activity following the manufacturer's instructions. Each set of experiments was performed in triplicate and repeated a least three times.

Analysis of in vivo E2 induced SUMO-1 and Ubc9-dependent E3 ubiquitin ligase activity of BRCA1 in COS-1 cells. Briefly, COS -1 cells were plated at a density of $1 \times 10^{6}$ cells per $100-\mathrm{mm}$ tissue culture dishes. After $24 \mathrm{~h}$ cells were cultured under sterol-free conditions in phenol-red-free medium (Life Technology) containing 5\% charcoal-stripped fetal bovine serum for $48 \mathrm{~h}$. Cells were transfected with $7 \mu \mathrm{g}$ of plasmid DNA (total of $21 \mu \mathrm{g}$ DNA) using FuGENE 6 (Roche Molecular Biochemicals, Indianapolis, IN, USA) according to the manufacturer's protocol. After $8 \mathrm{~h}$, the cells were incubated with $100 \mathrm{nM}$ E2 for $24 \mathrm{~h}$. Cells were then harvested in modified RIPA buffer (50 mM Tris- $\mathrm{HCl}, \mathrm{pH} 7.8 ; 150 \mathrm{mM}$ $\mathrm{NaCl} ; 5$ mM EDTA; $0.5 \%$ Triton X-100; 0.5 Nonidet P-40; $0.1 \%$ sodium deoxycholate) with protease inhibitors in the presence of $20 \mathrm{mM}$ NEM (N-Ethylmalemide). Lysates were syringed on ice with a 22-gauge syringe and centrifuged to separate insoluble proteins. SDS sample buffer was added to lysates and loaded on a $4-20 \%$ gradient gel. Membrane was transferred onto a polyvinylidene diflouride membrane overnight and then blocked by incubation with 5\% BSA and $0.001 \%$ Tween-20 overnight. ER $\alpha$ was detected with mouse monoclonal anti ER $\alpha$ antibody (1:100 dilution) and secondary anti-mouse HRP-conjugated secondary antibody 1:4000 dilution for $1 \mathrm{~h}$ at room temperature. The membrane was washed and the results were analyzed using LAS-IMAGE reader 3000 .

\section{Results}

Identification of SUMO-1 modification consensus sequence in the amino-terminal region of BRCAl/la/b protein. Posttranslational modification of transcription factors by SUMO (small ubiquitin related modifier) regulates their activity, turnover, localization and interactions (32). A cursory look at the conserved amino-terminal sequence of BRCA1/1a/1b proteins revealed a consensus SUMO modification site (Table I and Fig. 1). SUMO modification usually occurs on the consensus $\mathrm{K}$ (lysine) residue in $\Psi \mathrm{KXE} \mathrm{CS}$, which mediate binding to Ubc9 (ubiquitin-conjugating enzyme), the SUMO E2 conjugating enzyme. To study the functional relevance of the presence of SUMO-CS in BRCA1 proteins, we assayed for the ability of Ubc9 to bind to full length wild-type BRCA1/1a/1b, as well as Mutant \#1 (lysine 109 has been converted to Arginine) proteins by GST pull down assays. Ubc9 or GST alone as a control was immobilized on glutathione-sepharose beads and incubated with ${ }^{35} \mathrm{~S}$ methionine-labeled proteins produced by in vitro translation using rabbit reticulocyte lysates. Bound proteins were eluted with SDS sample buffer and analyzed by $8 \%$ SDS-PAGE. Full length BRCA1, BRCA1a and BRCA1b unlike Mutant \#1, bound specifically with Ubc9 suggesting that the lysine residue at 109 is critical for Ubc9 binding (Fig. 2a-c).

BRCA1 RING domain cancer-predisposing mutation C61G disrupts Ubc9 binding. BRCA1 C61G mutation observed in breast cancer patients has lost both BRCA1/BARD ubiquitin ligase activity as well as ability to inhibit ER $\alpha$ activity in breast cancer cells (33). We did not observe any significant binding of in vitro translated full length BRCA1 Mutant \#4 (C61G) to Ubc9 using GST capture assays (Fig. 2a). These results suggest a potential link between the loss of BRCA1 ubiquitin ligase activity, loss of ER $\alpha$ repression and Ubc9 binding.

A 182-amino acid domain near the amino terminus of BRCA1/la/lb is sufficient for binding to Ubc9 and ERa. We have found full length BRCA1/1a/1b proteins to inter- 
act in vitro with Ubc9 and since the N-terminal exons 2-8 (amino acid residues 1-182) are conserved among all these BRCA1 isoforms, we decided to study the interaction of BRCA1 amino acid 1-182 with Ubc9. Similarly since this region also binds in vitro to $\mathrm{ER} \alpha$ aa $338-379$ we studied the in vitro interaction of BRCA1 1-182 with $\mathrm{ER} \alpha 338-379$ using GST pull down assay described above. We observed binding of in vitro translated BRCA1 1-182 but not BRCA1 1-182 Mutant \#1 and Mutant \#4 (C61G) to Ubc9 and ERa 338-379 (Fig. 2d and e). The faint bands observed are due to nonspecific binding since they are also present in the GST lanes (Fig. 2e lanes 2, 5 and 6). These results indicate that the N-terminus of BRCA1 (aa 1-182) is sufficient for binding to both Ubc9 and ER $\alpha$. It also suggests the requirement of an intact RING domain and lysine 109 SUMO-acceptor sites for binding to Ubc9 and ER $\alpha$ in vitro. Mutant \#4 (C61G) and Mut\#1 showed auto-ubiquitination implying that the RING domain as well as lysine 109 SUMO-acceptor site is dispensable for this activity.

Ubc9 and SUMO defective mutant Ubc9 C93S compete in vitro with ERa (aa 338-379) for binding to BRCAl (aa 1-182). The requirement of the same region of BRCA1 (aa 1-182) for binding to both Ubc9/Ubc9C93S and ER $\alpha$ (aa 338-379) raises the possibility that Ubc9/Ubc9C93S and ER $\alpha$ (338-379) may compete for binding to BRCA1. We therefore performed $\mathrm{ER} \alpha$ (338-379) pull down experiments with BRCA1 (1-182). We found that on pre-incubation with increasing concentrations of Ubc9 or Ubc9 C93S there was decreased BRCA1 binding to ER $\alpha$ (338-379) (Fig. 2f and g). These results suggest that both Ubc9 and Ubc9 C93S have the ability to inhibit BRCA1 interaction with $\mathrm{ER} \alpha$ thereby antagonizing BRCA1 repression of $\mathrm{ER} \alpha$ activity.

Co-localization of endogenous and exogenous BRCA1/1a proteins with Ubc9 in human breast cancer cells. MCF-7 cells contain endogenous BRCA1 and Ubc9 proteins. To study the in vivo association of BRCA1 with Ubc9, immunofluorescence microscopy was performed using BRCA1 and Ubc9 antibodies. BRCA1 showed both nuclear as well as cytoplasmic localization, whereas the subcellular localization of Ubc9 was mostly nuclear. There was strong co-localization of BRCA1 and Ubc9 (Fig. 2h) in the merged images (yellow color). These results suggest that some BRCA1 and Ubc9 proteins do interact in vivo in breast cancer cells even in the absence of E2. We repeated these experiments using MCF7 cells that have been transfected separately with EGFPBRCA1 or EGFP-BRCA1a. We once again observed a clear co-localization of BRCA1 (Fig. 2i) as well as BRCA1a (Fig. 2j) with endogenous Ubc9 proteins. These results further support the in vivo interaction of BRCA1 proteins with Ubc9.

In vivo association of BRCAla but not BRCAla Mut\#1 with $U b c 9$ is ligand-dependent. Having previously shown that the BRCA1a protein interacts with Ubc9 in vivo in MCF-7 cells, we tested both the wild-type BRCA1a and mutant BRCA1a Mut\#1 to associate with Ubc9 in vivo, by IP-Western blot analysis (Fig. 2k). We have used BRCA1a in many of our experiments since it expressed at higher levels than BRCA1. The IP-Western blot assays showed that BRCA1a and
BRCA1a Mut\#1 were expressed in Cos-1 cells and anti-Flag IP of cells transfected with FLAG-BRCA1a in presence of E2 brought down significant amount of Ubc9 as compared with FLAG-IP of BRCA1a Mut\#1 (Fig. 2k, lanes 4 and 8). These results suggest that loss of BRCA1a Mut\#1 to repress E2-induced $\mathrm{ER} \alpha$ activity can be due to loss of its capacity to associate with Ubc9.

BRCA1/1a/1b but not Mutant \#1 represses E2-induced ERa activity in human breast cancer cells. Full length BRCA1 inhibits E2-induced ER $\alpha$ activity in breast cancer cells (34). Here, we tested the ability of full length BRCA1, 1a, 1b and their respective Mutant \#1 to inhibit E2-induced ER $\alpha$ activity using an ERE-luciferase reporter (35) containing the vitellogenin A2 ERE in plasmid pGL2 as described earlier (34). In T47D ER $\alpha$-positive breast cancer cells BRCA1/1a/1b significantly inhibited ligand induced ER $\alpha$ activity however BRCA1/1a Mutant \#1 failed to repress ER $\alpha$ activity (Fig. 3a). Consistent with the GST capture assays, Mutant \#1 which is unable to bind both Ubc9 as well as ER $\alpha$ 338-379 also lost its ability to inhibit ER $\alpha$ activity. These results suggest that the SUMO-acceptor site at lysine 109 which facilitates binding to both Ubc9 and ER $\alpha$ is required for repressing E2-indued $\mathrm{ER} \alpha$ activity in breast cancer cells. Thus based on our findings we can hypothesize the presence of an ER $\alpha$ regulatory switch in the amino terminal region of BRCA1 proteins which we have named as BRUERS (BRCA1 Ubc9 binding ER $\alpha$ regulatory switch, Fig. 1b). Mutation or post-translational modification of lysine in this sequence which disrupts the interaction to two cancer promoting proteins namely Ubc9 and ER $\alpha$ results in abrogation of $\mathrm{ER} \alpha$ repression leading to breast cancer.

Ubc9 but not Ubc9C93S represses E2-induced ERa mediated transcription by SUMO-1 in human breast cancer cells. ER $\alpha$ is a nuclear transcriptional factor that undergoes various types of post-translational modifications such as phosphorylation, acetylation, ubiquitination and sumoylation which regulates its activity. SUMO-1 has been shown to stimulate E2induced ER $\alpha$ transcription activity of the ERE-luciferase reporter in a dose-dependent manner (22). To examine the role of Ubc9 and SUMO-defective mutant Ubc9 C93S on E2 induced ER $\alpha$ transcription mediated by SUMO-1 using EREluciferase reporter plasmid in T47D breast cancer cells; we co-expressed Ubc9 or Ubc9 C93S along with SUMO-1. When SUMO-1 was co-expressed with Ubc9, the SUMO-1 mediated activation of ER $\alpha$ was strongly repressed (Fig. 3b, compare lane 2 with lane 3 ). Similar results were observed previously in HeLa cells (22) suggesting that expression of both SUMO-1 and Ubc9 results in extensive sumoylation of ER $\alpha$ and/or its co-factors that may be responsible for the repression of ER $\alpha$ activity (22). This repression of ER $\alpha$ activity was not significant when we used Ubc9C93S, a dominant negative mutant of Ubc9 further suggesting the involvement of sumoylation in repression of ER $\alpha$ activity (Fig. 3b, lanes 2 and 4). The expression of BRCA1a along with SUMO-1 weakly activated the E2-dependent ER $\alpha$ transcription (Fig. 3b, compare lanes 2 and 5).

BRCAla unlike Mut\#1 was able to relieve the hormonedependent ERa repression by Ubc9 in human breast cancer 


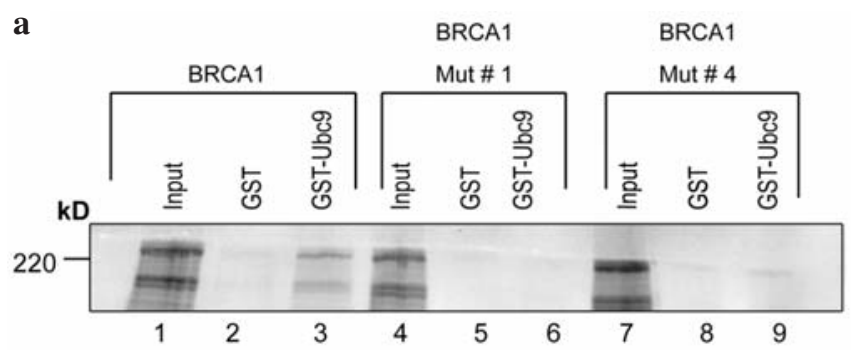

c
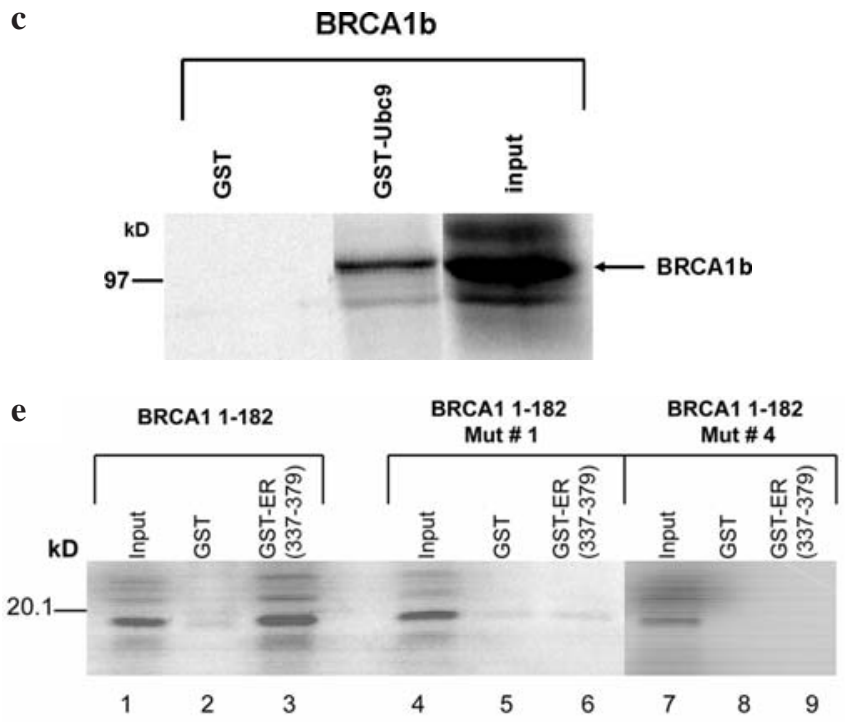

$\mathbf{g}$

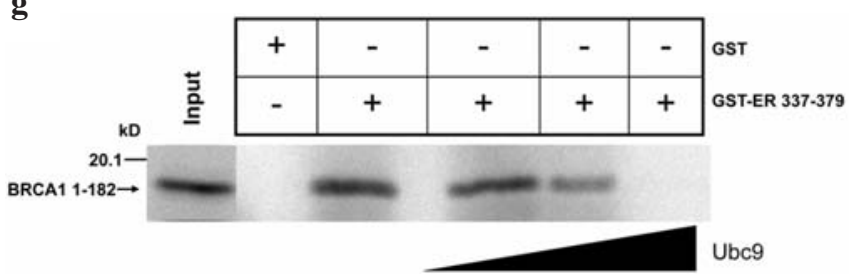

cells. The expression of BRCA1a unlike BRCA1a Mutant \#1 relieved the E2-dependent $\mathrm{ER} \alpha$ transcription repression by Ubc9 in T47D breast cancers cells (Fig. 3b, compare lanes 3 to 6 and 8). These results suggest BRCA1 proteins to antagonize SUMO-1 and Ubc9-dependent transcription repression of $\mathrm{ER} \alpha$ in T47D breast cancer cells.

BRCA1/1a/1b and BRCA1 (aa 1-182) but not Mut \#1 and Mut \#4 represses E2-induced ERa activity by catalytically inactive Ubc9 in human breast cancer cells. Ubc9 was found to be expressed at elevated levels in several metastatic cancers (36). Ubc9 has been shown previously to regulate ER $\alpha$ transcription independent of its sumoylation activity (22). We therefore studied the effect of BRCA1, BRCA1a, BRCA1b, BRCA1/1a Mutant \#1, BRCA1/1a Mut\#4, BRCA1 (1-182), BRCA1 (1-182) Mutant \#1 and Mut\#4 on E2-induced ER $\alpha$ activation by a catalytically inactive form of Ubc9, Ubc9/C 93S using an ERE-luciferase reporter in T47D breast cancer cells. We found BRCA1/1a (Fig. 3c, lanes 4 and 5; Fig. 3d, left panel, lanes 2 and 3) and BRCA1 (1-182) (Fig. 3d, right panel, lanes 2 and 3) unlike their respective Mut \#1 (Fig. 3c, lanes 5 and 6; Fig. 3d, left panel, lanes 3 and 4; Fig. 3c, right

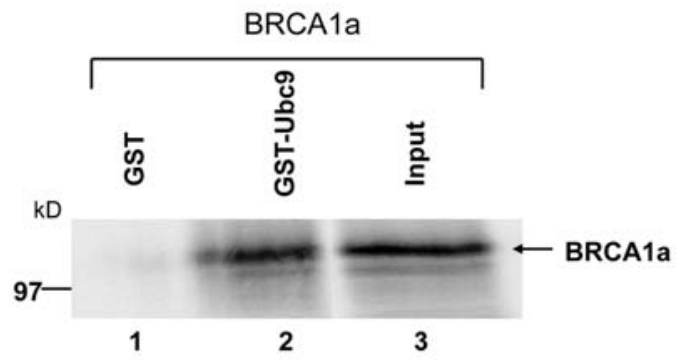

d
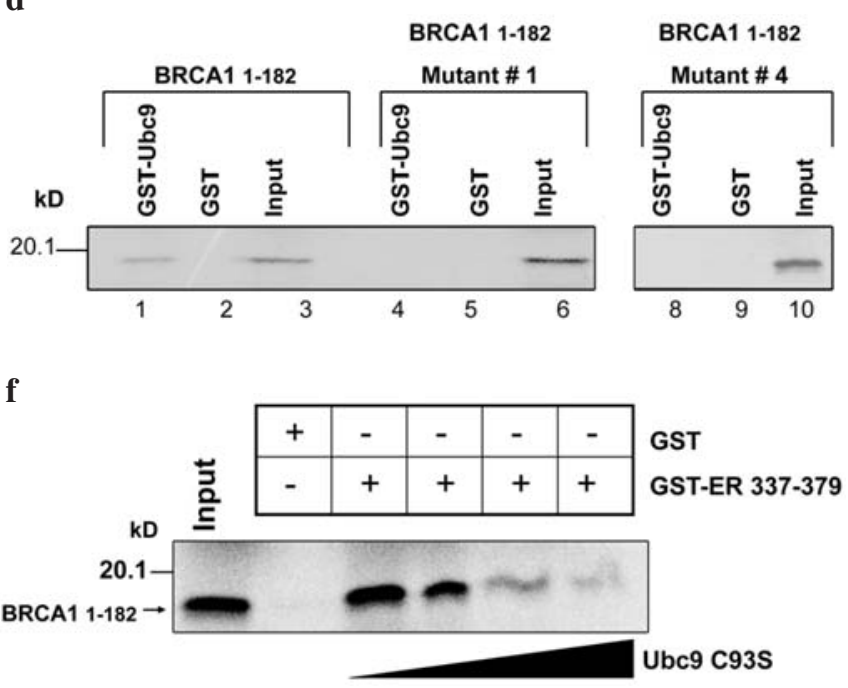

Figure 2. In vitro and in vivo binding of full length BRCA1/1a/1b/BRCA1 1-182 but not Mut\#1 and Mut\#4 to Ubc9 and ER $\alpha$ (aa 337-339). (a) In vitro binding of BRCA1 unlike Mut\#1 and Mut\#4 to Ubc9. (b) In vitro binding of BRCA1a to Ubc9. (c) In vitro binding of BRCA1b to Ubc9. (d) In vitro binding of BRCA1 1-182 but not Mut\#1 and Mut\#4 to Ubc9. (e) In vitro binding of BRCA1 1-182 but not Mut\#1 and Mut\#4 to ER $\alpha$ (aa 337-379). All the pull-downs were done at least 3 times. (f) Ubc9 C93S competes with $\mathrm{ER} \alpha$ for binding to BRCA1 1-182. (g) Ubc9 competes with $\mathrm{ER} \alpha$ for binding to BRCA1 1-182.

panel, lanes 3 and 4) and Mut\#4 (Fig. 3d, left panel, lanes 3 and 5; Fig. 3d, right panel, lanes 3 and 5) inhibited strongly E2-mediated ER $\alpha$ transcription activation by $\mathrm{Ubc} 9 / \mathrm{C} 93 \mathrm{~S}$ in breast cancer cells. Interestingly, BRCA1 1-182 Mut\#1 stimulated E2-induced ER $\alpha$ activity more than the control UbC93S in breast cancer cells (Fig. 3d compare lanes 2-4). These results indicate the requirement of SUMO-modification site in BRCA1 which interacts with Ubc9 in the repression of Ubc9/C93S mediated trans-activation of the ER $\alpha$.

ERa is degraded by the activation of E3 ubiquitin ligase activity of BRCAla but not BRCAla Mut \#1 only on association with Ubc9/Ubc9 C935 when co-expressed with $S U M O-1$. The ubiquitin pathway is important for degradation of $\mathrm{ER} \alpha$ and is also required for efficient $\mathrm{ER} \alpha$ trans-activation. The only known biochemical activity associated with BRCA1 is its E3 ubiquitin ligase activity (37). BRCA1 and the BARD1 proteins form a complex with the E2-conjugating enzyme $\mathrm{UbcH} 5 \mathrm{c}$ that can catalyze this activity in vivo. Recently ER $\alpha$ has been identified as a putative substrate for the BRCA1/ BARD1 ubiquitin ligase. Cancer pre-disposing mutations within the RING domain of BRCA1 were found to impair the 

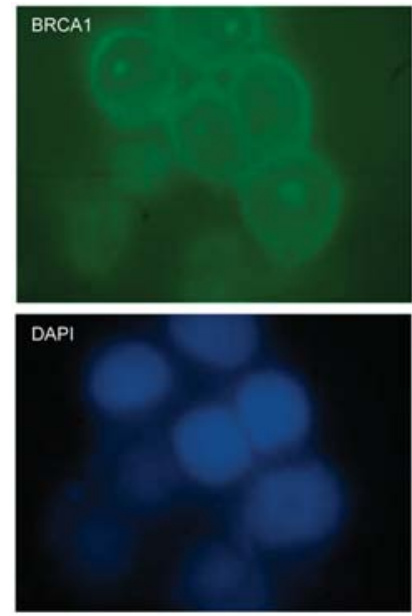

$\mathbf{j}$
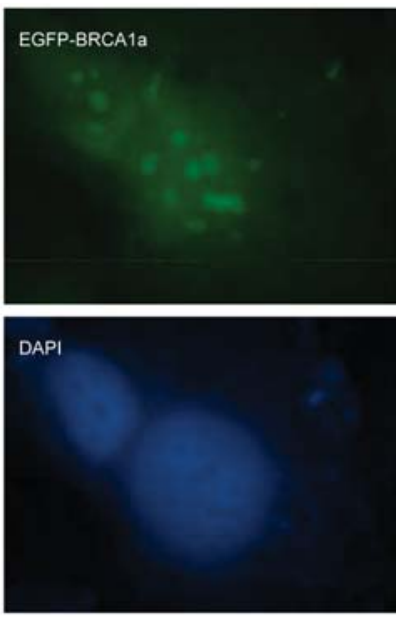
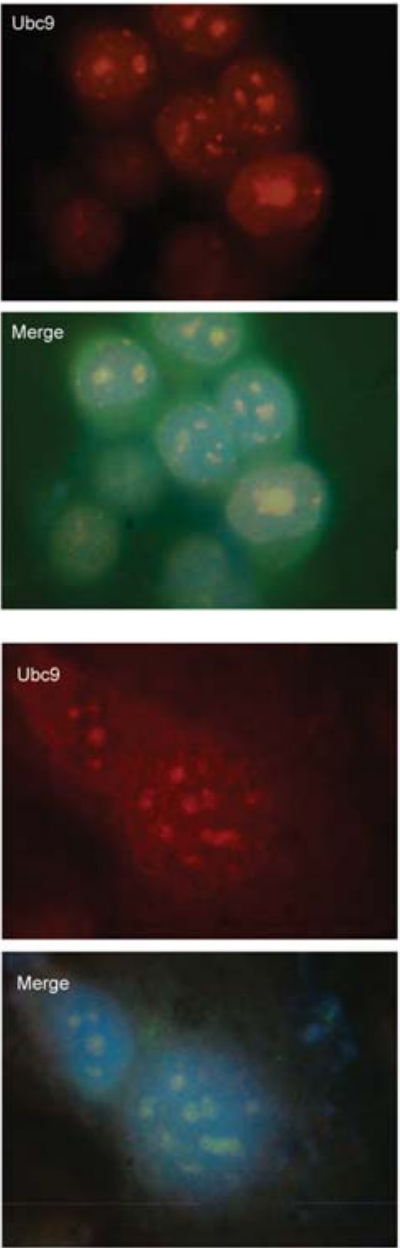
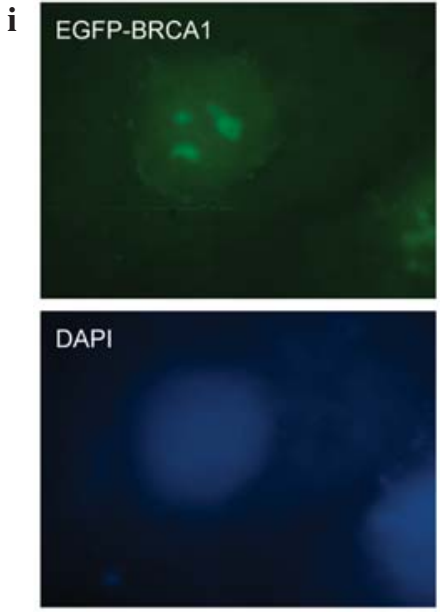

UBC9
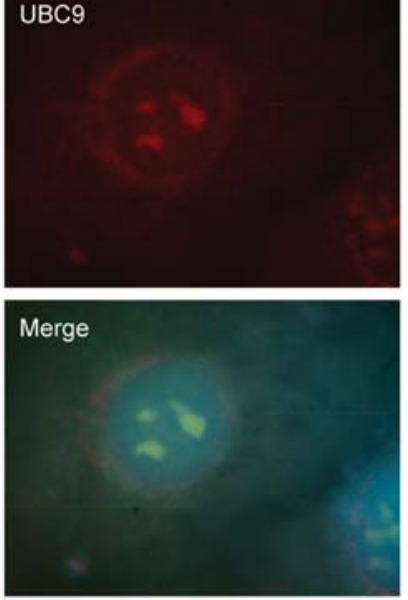

$\mathbf{k}$

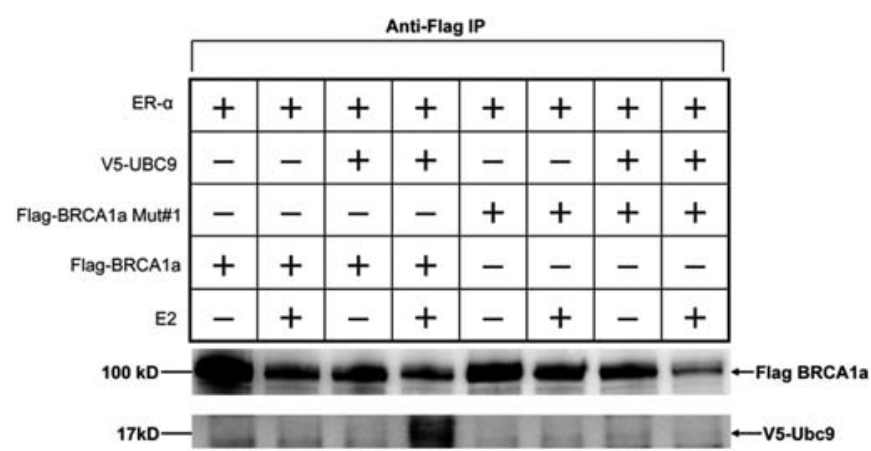

Figure 2 (continued). (h) Co-localization of endogenous BRCA1 proteins with Ubc9 in vivo in breast cancer cells. The endogenous BRCA1 protein expressed in MCF-7 cells was detected by immunostaining with anti-Ab-1 (EMD Chemicals) antibody and endogenous Ubc9 were detected by immunostaining with anti-Ubc9 (sc-10759, Santa Cruz) antibody. DAPI staining is shown in parallel. (i) Co-localization of full length EGFP-BRCA1 protein with Ubc9 in vivo in breast cancer cells. The GFP tagged BRCA1 was transiently expressed in MCF-7. The tagged protein was detected by immunostaining with anti-GFP (sc-9996, Santa Cruz) antibody. Endogenous Ubc9 was detected by immunostaining with anti-Ubc9 (sc-10759, Santa Cruz) antibody. DAPI staining is shown in parallel. (j) Co-localization of full length EGFP-BRCA1a protein with Ubc9 in vivo in breast cancer cells. Experimental conditions were similar to (i). (k) In vivo association of BRCA1a but not BRCA1a Mut \#1 with Ubc9 in presence of E2 in Cos-1 cells. Cos-1 cells were transiently transfected with UBC9 and ER $\alpha$ and FLAG-tagged BRCA1a or FLAG-tagged BRCA1a Mutant \#1 for 48 h. The transfected cell lysates were immunoprecipitated with anti-Flag M2 Affinity Gel, and the precipitated proteins were subjected to Western blotting to detect the FLAG and UBC9 proteins.

BRCA1/BARD1 ER $\alpha$ ubiquitination (30) as well as ER $\alpha$ transcriptional repression suggesting a link between loss of BRCA1 ligase activity and BRCA1-associated tissue-specific breast and ovarian cancers. Ubc9 was initially linked to ubiquitination due to its homology with ubiquitin-conjugated enzymes. The addition of purified human Ubc9 resulted in in vitro ubiquitination of activating transcription factor 2 (38). Recent results have found Ubc9 to be involved in sumoylation of several target proteins. Since BRCA1 is an E3-ubiquitin ligase it is pertinent to understand if its ability to relieve the transcriptional repression of $\mathrm{ER} \alpha$ is due to degradation of ER $\alpha$. ER $\alpha$ has been shown to get sumoylated by SUMO-1 and Ubc9 in vivo in Cos-1 cells (22). We therefore analyzed the ER $\alpha$ protein expression in E2-induced Cos-7 cells that were transiently transfected with constructs encoding ER $\alpha$, SUMO-1 Ubc9 or Ubc9/C93S in the absence and presence of BRCA1a or BRCA1a Mutant \#1. Transfected cells were lysed in the presence of N-ethylmaleimide (NEM) an inhibitor of SUMO-1 hydrolase and subjected to Western blot analysis using ER $\alpha$ antibody. As shown in Fig. 4, antiER $\alpha$ antibody detected a major band with mobility higher than the ER $\alpha$ band (Fig. 4, lane 1) which corresponds to sumoylated ER $\alpha$ (Fig. 4, lane 2). The ER $\alpha$ band almost disappeared in Cos-7 cells transfected with SUMO-1, Ubc9 and BRCA1a (Fig. 4, lane 6) and this band was faint in presence of Ubc9/C93S mutant (Fig. 4, lane 7). No such ligand-induced, Ubc9 or Ubc9/C93S mediated ER $\alpha$ degradation was seen in the presence of Mutant \#1 (Fig. 4, lanes 8 and 9) or BRCA1a and SUMO-1 alone (Fig. 4, lane 5). We have used BRCA1a in this study because it was found to be more stable compared to BRCA1 as well as BRCA1 1-182 (Rao, unpublished data). Similar results were obtained with BRCA1 1-182 but this protein had high auto ubiquitination activity and was unstable (data not shown). These results suggest a novel mechanism where the E3-ubiquitin ligase BRCA1, binds and recruits E2 SUMO-conjugating enzyme Ubc9 to ER $\alpha$ and facilitates its ubiquitination and proteosomedependent degradation in an estrogen-dependent manner. 


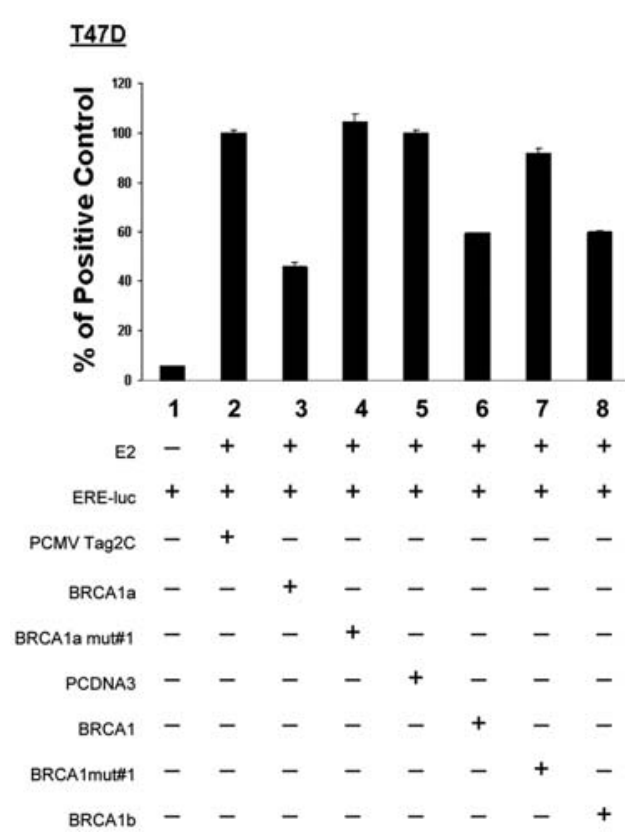

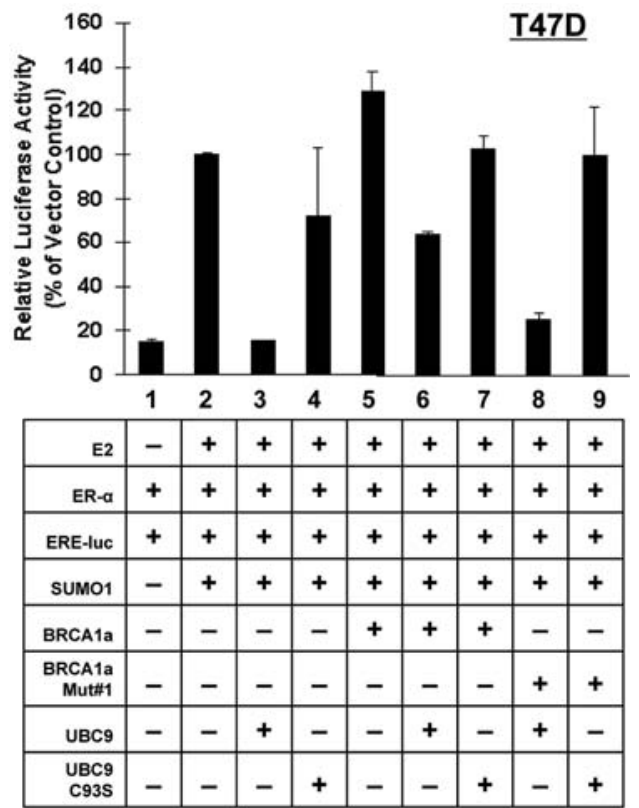

c

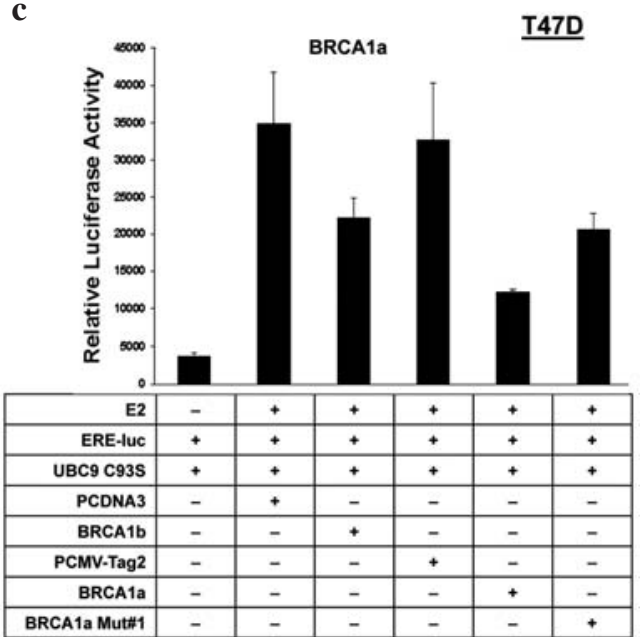

d
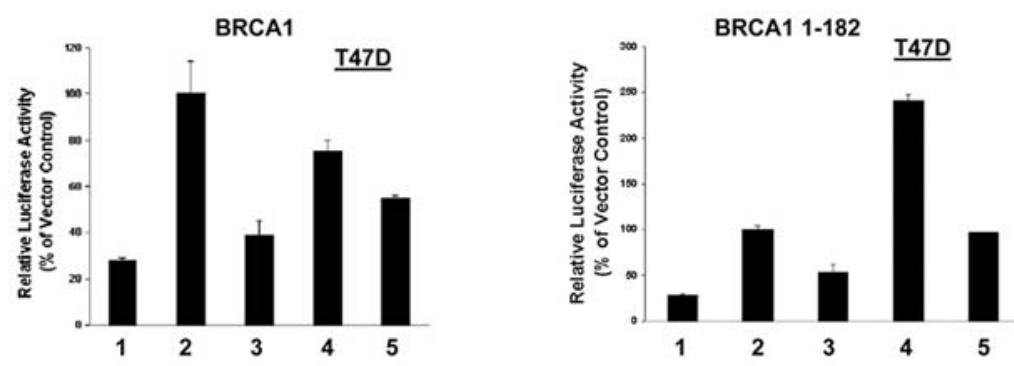

$47 \mathrm{D}$

Figure 3. BRCA1/1a/1b and BRCA1 1-182 but not Mut\#1 and Mut\#4 regulate E2-induced ER $\alpha$ activity in T47D breast cancer cells. (a) BRCA1/1a/1b but not Mut\#1 represses E2-induced ER $\alpha$ activity in breast cancer cells. T47D cells were transfected with ERE-luciferase reporter and WT BRCA1, BRCA1a, BRCA1b, BRCA1a Mutant \#1 or BRCA1 Mutant \#1 as indicated. After recovery, cells were treated with E2 $(1 \mu \mathrm{M})$ or ethanol vehicle (-). pCMV Tag2 and PCDNA3 vectors were also included for normalization of the transfections. Forty-eight hours after transfection, luciferase activity was analyzed by using a luciferase assay kit. The data are presented as the mean \pm SD of three independent experiments performed in triplicate. (b) BRCA1a, but not Mut\#1 relieve ER $\alpha$ repression by Ubc9 in breast cancer cells. T47D cells were transfected with ERE-luciferase reporter and SUMO-1 and BRCA1a, BRCA1a Mutant \#1 in presence of UBC9 or UBC9 C93S or without as indicated. After recovery, cells were treated with E2 $(1 \mu \mathrm{M})$ or ethanol vehicle (-). Forty-eight hours after transfection, luciferase activity was analyzed by using a luciferase assay kit. The data are presented as the mean \pm SD of three independent experiments performed in triplicate. (c) BRCA1a/1b but not Mut\#1 inhibit E2-induced ER $\alpha$ activity by Ubc9 C93S (SUMO-independent) in breast cancer cells. T47D cells were transfected with ERE-luciferase reporter and UBC9 C93S and BRCA1b, BRCA1a or BRCA1a Mut\#1 as indicated. After recovery, cells were treated with E2 $(1 \mu \mathrm{M})$ or ethanol vehicle (-). pCMV Tag2 and PCDNA3 vectors were also included for normalization of the transfections. Forty-eight hours after transfection, luciferase activity was analyzed by using a luciferase assay kit. The data are presented as the mean \pm SD of three independent experiments performed in triplicate. (d) BRCA1 and BRCA1 1-182 but not Mut\#1 and Mut\#4 inhibit E2-induced ER $\alpha$ activity by Ubc9 C93S (SUMO-independent) in breast cancer cells. T47D cells were transfected with ERE-luciferase reporter and UBC9 C93S and BRCA1, BRCA1 Mut\#1, BRCA1 Mut\#4, BRCA1 1-182, BRCA1 1-182 Mut\#1or BRCA1 1-182 Mut\#4 as indicated. After recovery, cells were treated with E2 (1 $\mu \mathrm{M})$ or ethanol vehicle (-). pCMV Tag2 and PCDNA3 vectors were also included for normalization of the transfections. Forty-eight hours after transfection, luciferase activity was analyzed by using a luciferase assay kit. The data are presented as the mean $\pm \mathrm{SD}$ of three independent experiments performed in triplicate.

This is the first time that an E2 SUMO-conjugating enzyme has been shown to participate in ubiquitination depending on the interacting partners. Ubc9 is a new player in E2-induced $\mathrm{ER} \alpha$ degradation by BRCA $1 / 1 \mathrm{a} / 1 \mathrm{~b}$ proteins in breast cancers. These results suggest a role for BRCA1 RING finger E3 ubiquitin ligase in bringing together ER $\alpha$ substrate and Ubc9 E2 enzyme to mediate the degradation of ER $\alpha$ via the ubiquitin-proteasome pathway in normal breast mammary cells and inhibition of this function due to lack of BRCA1 may prevent receptor turnover resulting in ER-negative 


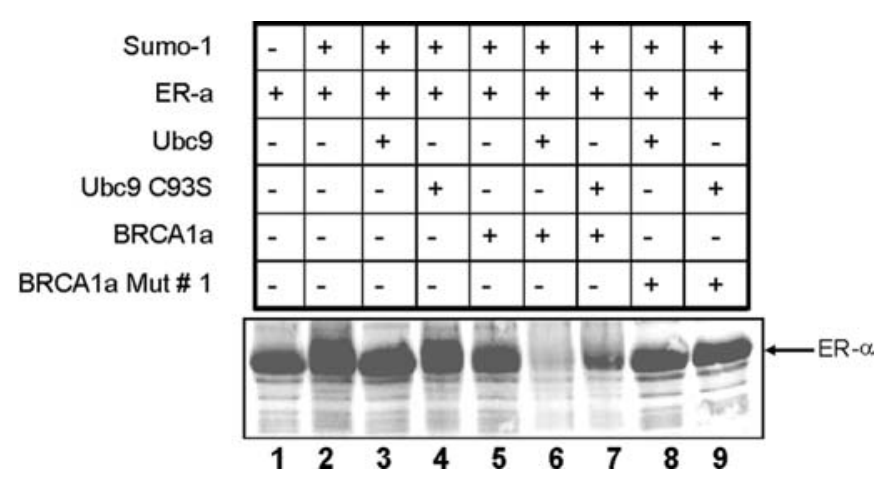

Figure 4. Degradation of E2-induced ER $\alpha$ by BRCA1a, but not Mut\#1 occurs only in the presence of Ubc9 or Ubc9C93S.

breast cancers. Since ER $\alpha$ was found to be degraded even in the presence of a sumo-defective mutant Ubc9 suggesting a sumoylation-independent function of Ubc9 in the degradation of $\mathrm{ER} \alpha$.

\section{Discussion}

Identifying the physiological function of BRCA1 in ER $\alpha$ signaling is key to understanding how BRCA1 dysfunction results in tissue-specific breast and ovarian cancers. In this report we have demonstrated for the first time the functional significance of the presence of a consensus SUMO modification site in the amino-terminal region of BRCA1, BRCA1a/1b proteins. Mutation in this potential SUMO modification site (K109 to R) impaired the ability of these proteins to both bind to $\mathrm{ER} \alpha$, Ubc9 as well as repress Ubc9 mediated SUMO-dependent/independent E2-induced ER $\alpha$ transcriptional activity in breast cancer cells. Furthermore, we have mapped BRCA1 (aa 1-182) which contains one of the SIM and RING-finger domain as the minimum domain that is sufficient for in vitro binding to Ubc9 as well as for regulating ER $\alpha$ activity. Interestingly, addition of BRCA1a but not Mut\#1 (K 109 to R) to E2-induced ER $\alpha$ resulted in degradation of ER $\alpha$ when co-expressed with SUMO-1 and Ubc9 both of which have been previously shown to sumoylate ER $\alpha$ suggesting BRCA1 to be a putative SUMO-1 and Ubc9dependent E3 ubiquitin ligase for ER $\alpha$. We therefore propose the term BRULERS1 (BRCA1 E3 ubiquitin ligase for ER $\alpha$ SUMO-1 and Ubc9 conjugate) as alternate name for this domain to highlight its function (Fig. 1b). The E3 ubiquitin ligase is the most important player in the ubiquitin-proteosome pathway. Infact aberrations in the E3 ubiquitin-protein ligase activity is involved in several human diseases (39). The observation that BRCA1-K109 to R does not interact with $\mathrm{ER} \alpha, \mathrm{Ubc} 9$, does not repress $\mathrm{ER} \alpha$ activity together with loss of degradation of ER $\alpha$ suggests that ubiquitination of $\mathrm{ER} \alpha$ by BRCA1 could regulate $\mathrm{ER} \alpha$ transcriptional activation. Ubc9 may have multiple functions on ER $\alpha$ like sumoylation of $\mathrm{ER} \alpha$, co-activator of $\mathrm{ER} \alpha$ and also ubiquitination of ER $\alpha$. Majority of BRCA1-related breast cancers are ER-negative and more prevalent in younger African American Women with breast cancer (40). Using a mouse model lacking BRCA1 it was found that the tumors were ER $\alpha$-positive at their early stages and later became ER $\alpha$-negative (41). The reason for this observation is unclear. We can speculate that lack of BRCA1 results in ER-negative tumors due to sumoylation of $\mathrm{ER} \alpha$ and its cofactors resulting in transcriptional silencing of $\mathrm{ER} \alpha$ expression. BRCA1 proteins may be an integral part of the sumoylation machinery that function to regulate the
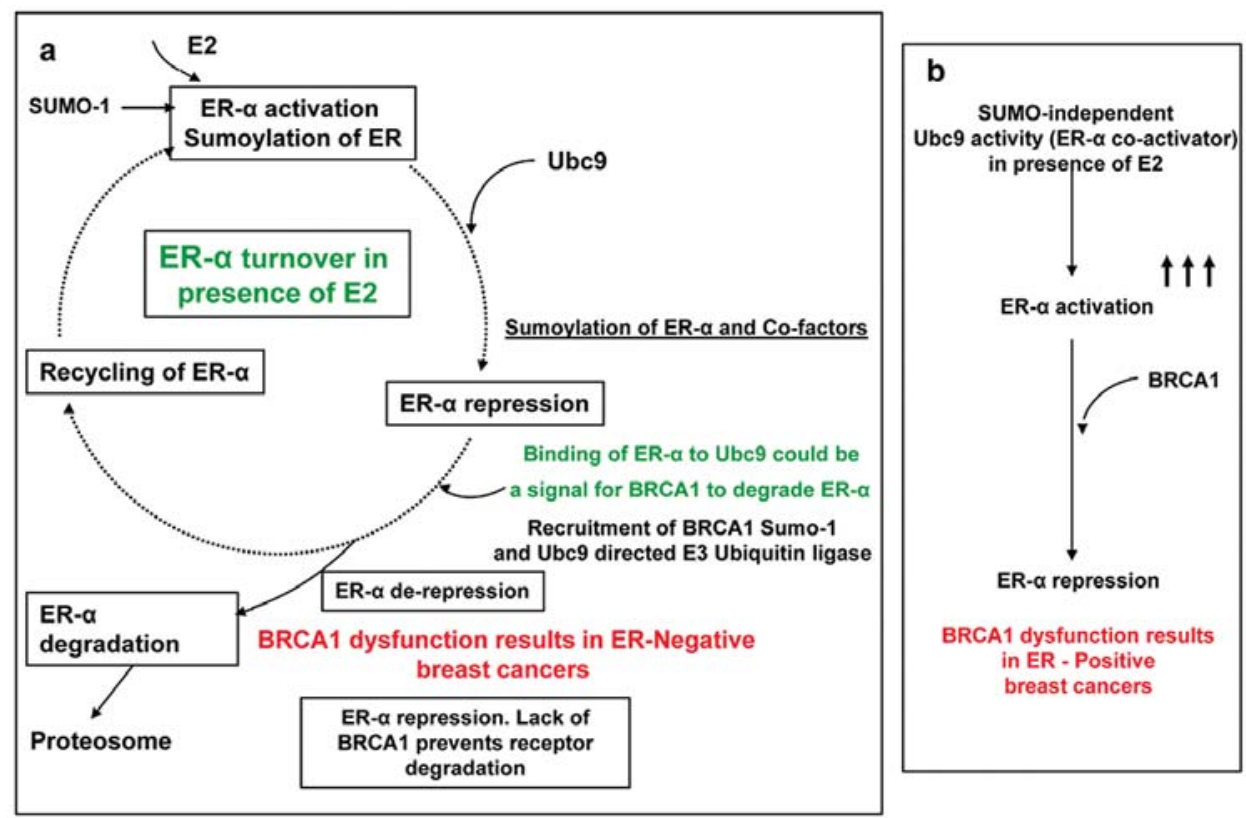

Figure 5. Models showing how BRCA1 dysfunction in regulating the E2-induced ER $\alpha$-activation/repression by SUMO-dependent/independent activities of Ubc9 results in: (a) ER $\alpha$-negative; (b) ER $\alpha$-positive breast cancers. 
overall sumoylation activity of ER $\alpha$ activity in breast and ovarian cancer cells similar to the RNF4 family of RINGfinger E3 ubiquitin ligase (42). We can hypothesize that BRCA1 belongs to the family of SIM-containing RINGfinger proteins (43) that induce turnover of $E R \alpha$ following its sumoylation. Based on these results we have proposed models showing how BRCA1 dysfunction in regulating ER $\alpha$ activity leads to ER $\alpha$-negative/positive breast cancers (Fig. 5). These studies also suggest that BRCA1-mediated ubiquitination of $\mathrm{ER} \alpha$ is tightly regulated in the cell. Alternately, it could be possible that SUMO modification followed by attachment of Ubc9 to ER $\alpha$ can serve as a signal for BRCA1 proteins to target it for degradation. There are currently no targeted treatments that are effective against TN breast cancers. The results from these studies uncover the paradox as to why BRCA1 dysfunction leads to triple-negative breast cancers, as well as develop novel targeted therapies based on enhancing the degradation of repressed ER $\alpha$ to reinitiate transcription offers a promising method for the treatment of these ER-negative breast cancers.

\section{Acknowledgements}

We thank Kartik Aysola and Vaishali Reddy for secretarial assistance. We thank Pat Abramson, DIT Morehouse School of Medicine for his help in formatting the figures. We thank all the other members of Rao and Reddy labs for their help. V.N. Rao dedicates this paper to her mother, Rohini N. Rao. This work was funded by Georgia Cancer Coalition Distinguished Cancer Scholar Awards to V.N. Rao and E.S.P. Reddy. This work was supported in part by NIHNCRR-RCMI grant G-12-RR03034.

\section{References}

1. Miki Y, Swensen J, Shattuck-Eidens D, Futreal PA, Harshman K and Tavtigian S: A strong candidate for the breast and ovarian cancer susceptibility gene BRCA1. Science 266: 66-71, 1994.

2. Rowell S, Newman B, Boyd J and King MC: Inherited predisposition to breast and ovarian cancer. Am J Hum Genet 55: 861-865, 1994.

3. Gayther SA, Warren W, Mazoyer S, Russell PA, Harrington PA, Chiano M, et al: Germline mutations of the BRCA1 gene in breast and ovarian cancer families provide evidence for a genotypephenotype correlation. Nat Genet 11: 428-433, 1995.

4. Orban TI and Olah E: Emerging roles of BRCA1 alternative splicing. Mol Pathol 56: 191-197, 2003.

5. Thakur S, Zhang HB, Peng Y, Le H, Carroll B, Ward T, et al: Localization of BRCA1 and a splice variant identifies the nuclear localization signal. Mol Cell Biol 17: 444-452, 1997.

6. Wilson CA, Payton MN, Elliott ES, Buaas FW, Cajulis EE, Grosshans D, et al: Differential subcellular localization, expression and biological toxicity of BRCA1 and the splice variant BRCA1-delta 11b. Oncogene 14: 1-16, 1997.

7. Cui JQ, Shao N, Chai Y, Wang H, Reddy ES and Rao VN: BRCAl splice variants BRCAla and BRCAlb associate with CBP co-activator. Oncol Rep 5: 591-595, 1998.

8. Wang H, Shao N, Ding OM, Cui J, Reddy ESP and Rao VN: BRCA1 proteins are transported to the nucleus in the absence of serum and splice variants BRCA1a, BRCA $1 \mathrm{~b}$ are tyrosine phosphoprotein that associate with E2F, cyclins and cyclin dependent kinases. Oncogene 15: 143-157, 1997.

9. Lu M, Conzen SD, Cole CN and Arrick B: Characterizations of functional messenger RNA splice variants of BRCA1 expressed in non malignant and tumor-derived breast cells. Cancer Res 56: 4578-4581, 1996.

10. Orban TI and Olah E: Expression profiles of BRCA1 splice variants in a synchronous and in G1/S synchronized tumor cell lines. Biochem Biophys Res Commun 280: 32-38, 2001.
11. Rosen EM, Fan S and Ma Y: BRCA1 regulation of transcription. Cancer Lett 236: 175-185, 2006.

12. Park MA, Seok Y, Jeong G and Lee J: SUMO1 negatively regulates BRCA1-medicated transcription, via modulation of promoter occupancy. Nucleic Acids Res 36: 263-283, 2008.

13. Foulkes WD, Metcalfe K, Sun P, Hanna WM, Lynch HT, Ghadirian $\mathrm{P}$, et al: Estrogen receptor status in BRCA1-and BRCA2-related breast cancer: the influence of age, grade, and histological type. Clin Cancer Res 10: 2029-2034, 2004.

14. Morris GJ, Naidu S, Topham AK, Guiles F, Xu Y, McCue P, et al: Differences in breast carcinoma characteristics in newly diagnosed African-American and Caucasian patients: a singleinstitution compilation compared with the National Cancer Institute's Surveillance, Epidemiology, and End Results Database. Cancer 110: 876-884, 2007.

15. Sorlie T, Tibshirani R, Parker J, Hastie T, Marron JS, Nobel A, et al: Repeated observation of breast tumor subtypes in independent gene expression data sets. Proc Natl Acad Sci USA 100: 8418-8423, 2003.

16. Turner NC and Reis-Filho JS: Basal-like breast cancer and the BRCA1 phenotype. Oncogene 25: 5846-5853, 2006.

17. Chai Y, Shao N, Lee L, Reddy V, Gabriela O, Rao R, et al: BRCA1a has antitumor activity in Triple-negative breast and ovarian and prostate cancer cells. Oncogene 26: 6031-6037, 2007.

18. Rebbeck TR, Levin AM, Eisen A, Snyder C, Watson P, Cannon-Albright L, et al: Breast cancer risk after bilateral prophylactic oophorectomy in BRCA1 mutation carriers. J Natl Cancer Inst 91: 1475-1479, 1999.

19. Ma YX, Tomita Y, Fan S, Wu K, Tong Y, Zhao Z, et al: Structural determinants of the BRCA1: estrogen receptor interaction. Oncogene 24: 1831-1846, 2005.

20. Song J, Zhang Z, Hu W and Chen Y: Small ubiquitin-like modifier (SUMO) recognition of a SUMO binding motif; a reversal of the bound orientation. J Biol Chem 280: 40122-40129, 2005.

21. Rodriguez, MS, Dargemont C and Hay RT: SUMO-1 conjugation in vivo requires both a consensus modification motif and nuclear targeting. J Biol Chem 276: 12654-12659, 2001.

22. Sentis S, Romancer M L, Bianchin C, Rostan M and Corbo L: Sumoylation of the estrogen receptor $\alpha$ Hinge region regulates its transcriptional activity. Mol Endocr 19: 2671-2684, 2005.

23. Kobayashi S, Shibata H, Yokota K, Suda N, Murai A, Kurihara I, et al: FHL2, UBC9 and PIASI are novel estrogen receptor and interacting proteins. Endocr Res 30: 617-621, 2004.

24. Lonard DM, Nawaz Z, Smith CL and O'Malley BW: The 26S proteasome is required for estrogen receptor- $\alpha$ and coactivator turnover and for efficient estrogen receptor- $\alpha$ transactivation. Mol Cell 5: 939-948, 2000.

25. Koonin E, Altschul SF and Bork P: BRCA1 protein products functional motifs. Nat Genet 13: 266-268, 1996.

26. Bork P, Hofmann K. Bucher P, Neuwald AF, Altschul SF and Koonin EV: A super family of conserved domains in DNA damage-responsive cell cycle checkpoint proteins. FASEB J 11: 68-76, 1997.

27. Nilsson S, Makela S, Treuter E, Tujague M, Thomsen J, Andersson $\mathrm{G}$, et al: Mechanisms of estrogen action. Physiol Rev 81: 1535-1565, 2001 .

28. Luo M, Koh M, Feng J, Wu Q and Melamed P: Cross talk in hormonally regulated gene transcription through induction of estrogen receptor ubiquitylation. Mol Cell Biol 25: 7386-7398, 2005.

29. Eakin CM, MacCoss MJ, Finney GL and Klevit RE: Estrogen receptor $\alpha$ is a putative substrare for the BRCA1 ubiquitin ligase. Proc Natl Acad Sci USA 104: 5794-5799, 2007.

30. Hashizume R, Fukuda M, Maeda I, Nishikawa H, Oyake D, Yabuki Y, et al: The RING heterodimer BRCA1-BARD1 is a ubiquitin ligase inactivated by a breast cancer derived mutation. J Biol Chem 276: 14537-14540, 2001.

31. Chai YL, Cui J, Chipitsyna G, Liao B, Lui S, Aysola K, et al: c-Fos oncogene regulator Elk-1 interacts with BRCA1 splice variants BRCA1a/1b and enhances BRCA1a/1b mediated growth suppression in breast cancer cells. Oncogene 20: 1357-1367, 2001.

32. Hay RT: SUMO: a history of modification. Mol Cell 18: 1-12, 2005.

33. Fan S, Ma YX, Wang C, Yuan RQ, Meng Q, Wang JA, et al: Role of direct interaction in BRCA1 inhibition of estrogen receptor activity. Oncogene 20: 77-87, 2001.

34. Fan S, Wang J, Yuan R, Ma Y, Meng Q, Erdos MR, et al: BRCA1 inhibition of estrogen receptor signaling in transfected cells. Science 284: 1354-1356, 1999. 
35. Henttu P, Kalkhoven E and Parker G: AF-2 activity and recruitment of steroid receptor coactivator 1 to the estrogen receptor depend on a lysine residue conserved in nuclear receptors. Mol Cell Biol 17: 1832-1839, 1997.

36. Moschos SJ, Smith AP, Mandic M, Athanassiou C, WatsonHurst K, Jukic DM, et al: SAGE and antibody array analysis of melanoma-infiltrated lymph nodes: identification of Ubc9 as an important molecule in advanced-stage melanomas. Oncogene 26: 4216-4225, 2007

37. Ohta $\mathrm{T}$ and Fukuda M: Ubiquitin and breast cancer. Oncogene 23: 2079-2088, 2004.

38. Firestein R and Feuerstein N: Association of activating transcription factor 2 (ATF2) with the ubiquitin-conjugating enzyme hUBC9. Implication of the ubiquitin/proteasome pathway in regulation of ATF2 in T cells. J Biol Chem 273: 5892-5902, 1998.

39. Fang S, Lorick K, Jensen J and Weissman A: RING finger ubiquitin protein ligases: implications for tumorigenesis, metastasis and for molecular targets in cancer. Semin Cancer Biol 13: 1-14, 2002.
40. Tischkowitz MD and Foulkes WD: The basal phenotype of BRCA1-related breast cancer: past, present and future. Cell Cycle 5: 963-967, 2006.

41. Li W, Xiao C, Vonderhaar BK and Deng C-X: A role of estrogen/ $\mathrm{ER} \alpha$ signaling in BRCA1-associated tissue-specific tumor formation. Oncogene 26: 7204-7212, 2007.

42. Sun H, Leverson J and Hunter T: Conserved function of RNF4 family proteins in eukaryotes: targeting a ubiquitin ligase to SUMOylated proteins. EMBO J 26: 4102-4112, 2007.

43. Uzunova K, Gottsche K, Miteva M, Weisshaar S, Glanemann C, Schnellhardt M, Niessen M, et al: Ubiquitin-dependent proteolytic control of SUMO conjugates. J Biol Chem 282: 34167-34175, 2007. 